\title{
Evaluation of Four Antiseptics Using a Novel Murine Norovirus
}

\author{
Takashi MATSUHIRA ${ }^{1)}$, Chizuko KAJI ${ }^{1)}$, Shoichi MURAKAMI ${ }^{1)}$, Kazunori MAEBASHI ${ }^{1)}$, \\ Tomoichiro $\mathrm{OKA}^{2)}$, Naokazu TAKEDA ${ }^{3)}$, and Kazuhiko KATAYAMA ${ }^{2)}$
}

${ }^{1)}$ Pharmaceutical Research Center, Meiji Seika Pharma Co., Ltd., 760 Morooka-cho, Kohoku-ku, Yokohama 222-8567, 2)Department of Virology II, National Institute of Infectious Diseases, 4-7-1 Gakuen, Musashi-murayama, Tokyo 208-0011, Japan, and ${ }^{3)}$ Section of Viral Infections, Thailand-Japan Research Collaboration Center on Emerging and Re-Emerging Infections (RCC-ERI), 6th Fl., Building 10, Department of Medical Sciences, Ministry of Publlic Health, Tiwanon Rd., Muang, Nonthaburi 11000, Thailand

\begin{abstract}
We isolated a novel murine norovirus (MNV), MT30-2 strain, from feces of conventional mice in Japan to evaluate the virucidal activity of four antiseptics. The MNV MT30-2 strain was inactivated by as little as $0.2 \%(\mathrm{w} / \mathrm{v})$ povidone-iodine (PVP-I) and $0.1 \%$ $(\mathrm{w} / \mathrm{v})$ sodium hypochlorite $(\mathrm{NaOCl})$ treatment as determined by a novel plaque assay. Importantly, PVP-I reduced the MNV titer by $4 \log _{10}$ within $15 \mathrm{~s}$ of exposure. The other two antiseptics, benzethonium chloride (BEC) and chlorhexidine gluconate (CHG), did not reduce the MNV titer even when treatment lasted for $60 \mathrm{~s}$. When the virus titer was reduced by PVP-I or $\mathrm{NaOCl}$ treatment, the amount of MNV RNA was not reduced, indicating that the presence of viral RNA was not related to the virucidal activity of the antiseptics. PVP-I and $\mathrm{NaOCl}$ will be useful in controlling the spread of MNV, which is a common problem in mice colonies. In this study, we isolated a novel MNV and newly revealed that two antiseptics (PVP-I and $\mathrm{NaOCl}$ ) were able to inactivate MNV at low concentrations and in a short contact time.
\end{abstract}

Key words: antiseptics, murine norovirus, plaque assay, quantitative RT-PCR

\section{Introduction}

Noroviruses belong to a group of single-stranded RNA, nonenveloped viruses in the Caliciviridae family that are widely transmitted among humans or animals. At present, five major genogroups (GI-GV) of noroviruses have been designated, with GI, GII, and GIV infecting humans, GIII infecting bovines, and GV infecting murines [17]. Murine norovirus (MNV), prototype MNV-1, was first identified in 2003 in immunocompromised mice lacking recombination-activating gene 2
(RAG2), signal transducer, and activator of transcription 1 (STAT1) $\left(\mathrm{RAG}^{2} / \mathrm{STAT}^{-/-}\right.$) [9]. Human norovirus (HuNoV) cannot grow in cell culture [5]. On the other hand, $\mathrm{MNV}$ is the only norovirus capable of replicating in both cell culture and small animals $[17,18]$. Infection of MNV in a normal mouse shows no visible signs, but MNV can induce a clinical disease with a high mortality in congenitally immunodeficient mice [13]. The major infection route of MNV is a fecal-oral route, and shedding of MNV continues for at least eight weeks post infection [6-8]. Accordingly, MNV is one of the most

(Received 27 April 2011 / Accepted 25 July 2011)

Address corresponding: T. Matsuhira, Pharmaceutical Research Center, Meiji Seika Pharma Co., Ltd., 760 Morooka-cho, Kohoku-ku, Yokohama 222-8567, Japan

(C) 2012 Japanese Association for Laboratory Animal Science 
transmittable and prevalent pathogens in laboratory mice today $[6,8,12]$, making the prevention of the spread of MNV an important issue.

In a previous study using the MNV CW1 strain [18], the antiseptic effect of ethanol (EtOH) against MNV was examined [1], and 60\% (v/v) EtOH was shown to reduce the MNV infectious titer by $4 \log _{10}$ within $30 \mathrm{~s}$ of exposure; however, $30 \%$ (v/v) EtOH did not reduce the titer. Moreover, $1 \%(\mathrm{w} / \mathrm{v})$ povidone-iodine (PVP-I) and $0.26 \%$ $(\mathrm{w} / \mathrm{v})$ sodium hypochlorite $(\mathrm{NaOCl})$ have been shown to inactivate the MNV CW1 strain, although the efficacies of these antiseptics against other MNV strains are unknown.

In this study, to clarify the virucidal activity of antiseptics against another MNV strain, we isolated a novel MNV, strain MT30-2, from conventional mice in Japan. Using the MT30-2 strain, the virucidal activity of PVPI was examined. PVP-I has been used as an external treatment for humans and has a broad spectrum of virucidal activity against enveloped and nonenveloped DNA and RNA viruses [10]. We compared the virucidal activity of PVP-I with those of $\mathrm{NaOCl}$, benzethonium chloride $(\mathrm{BEC})$, and chlorhexidine gluconate $(\mathrm{CHG})$ using plaque assays. Furthermore, we quantified MNV MT302 RNA copies by quantitative reverse transcription polymerase chain reaction (RT-PCR) and compared the results with virus titers determined in the plaque assay.

\section{Materials and Methods}

\section{Mice}

Six-week-old male conventional ICR mice were purchased from a conventional Japanese mouse breeder (Chiba, Japan). The mice were kept in a temperature-and light-controlled environment with standard food and water given ad libitum. All experiments were approved by the Animal Care and Use Committee of Meiji Seika Pharma Pharmaceutical Research Center.

\section{Cells and virus infection}

RAW264.7 cells, a mouse macrophage cell line, were purchased from DS-Pharma (Osaka, Japan) and cultured in high-glucose Dulbecco's modified Eagle medium + GlutaMAX $^{\mathrm{TM}}$-I (DMEM; Invitrogen, Carlsbad, CA, USA) supplemented with $25 \mathrm{mM}$ HEPES and $10 \%$ fetal bovine serum (FBS) at $37^{\circ} \mathrm{C}$ in $5 \% \mathrm{CO}_{2}$. $\mathrm{MNV}$ was inoculated onto a monolayer of RAW264.7 cells, and cultured in DMEM supplemented with $25 \mathrm{mM}$ HEPES and $2 \% \mathrm{FBS}$ (DMEM-2\% FBS) at $37^{\circ} \mathrm{C}$ in $5 \% \mathrm{CO}_{2}$. Three to 5 days later, the cultured medium was centrifuged at $15,000 \mathrm{~g}$ for $5 \mathrm{~min}$ at $4^{\circ} \mathrm{C}$, and the supernatant was used as the virus stock.

\section{Isolation of $M N V$}

Two stool samples per mouse were collected, homogenized in PBS, and then centrifuged at 15,000 $g$ for 15 min at room temperature. The supernatant was passed through a $0.22-\mu \mathrm{m}$-pore-size filter, diluted in DMEM-2\% FBS, and used to inoculate RAW264.7 cells. Four days later, the culture medium and RAW264.7 cells were frozen at $-80^{\circ} \mathrm{C}$. After thawing, the culture medium and cells were centrifuged at $8,000 \mathrm{~g}$ for $5 \mathrm{~min}$ at $4^{\circ} \mathrm{C}$, and the supernatant was used for further passage. A total of nine passages followed by two successive plaque purifications were performed. Plaque assays were performed as previously described [18] with RAW264.7 cells.

\section{$M N V$ inactivation experiment}

Different antiseptics were used to inactivate the MNV. PVP-I (ISODINE solution 10\%) was obtained from Meiji Seika Pharma (Tokyo, Japan). $\mathrm{NaOCl}$ (Purelox-S) was purchased from Oyalox (Tokyo, Japan). BEC (Hyamine solution 10\%) was purchased from Daiichi Sankyo (Tokyo, Japan). CHG (Hibitane gluconate 20\%) was purchased from Dainippon Sumitomo Pharma (Osaka, Japan). Inactivation was induced by adding 100 $\mu 1$ of the diluted antiseptic [0.4 or $2 \%(\mathrm{w} / \mathrm{v})$ PVP-I, 0.2 or $0.4 \%$ (w/v) NaOCl, $0.2 \%$ (w/v) BEC, $1 \%$ (w/v) CHG] to $100 \mu \mathrm{l}$ of virus stock solution. The final volume of $200 \mu 1$ reaction mixture was incubated for $15,30,45$ or $60 \mathrm{~s}$ at room temperature prior to neutralization. Neutralization control samples were used as 0 -s incubation samples. To stop the effect of PVP-I, $2 \mu 1$ of $1 \mathrm{M}$ sodium thiosulfate was added to the reaction mixture. To stop the effect of $\mathrm{NaOCl}, 10 \mu \mathrm{l}$ of $1 \mathrm{M}$ sodium thiosulfate was added to the reaction mixture, followed by $10 \mu 1$ of 1 M HEPES (pH 7.4). The neutralized solutions obtained from the above treatment were then 10-fold diluted with DMEM-2\% FBS. For the neutralization control, PVP-I and $\mathrm{NaOCl}$ were first neutralized with sodium thiosulfate 
and diluted with DMEM-2\% FBS before being added to the virus stock. To stop the effect of BEC and CHG, the reaction mixtures were 100-fold diluted with DMEM-2\% FBS. The neutralization controls for BEC and CHG were prepared by diluting the virus stock with DMEM$2 \%$ FBS without adding antiseptics. The virus titer and copy numbers of MNV RNA in the reaction mixture were measured by plaque assays and quantitative RT-PCR, respectively.

\section{$R T-P C R$}

Viral RNA was extracted from the fecal suspension or supernatant using QIAamp Viral RNA Mini Kits (QIAGEN, Valencia, CA, USA) according to the manufacturer's instructions. An RT-PCR primer pair (forward, 5' -TTTGGAACAATGGATGCTGA-3'; reverse, 5'-TAGGGTGGTACAAGGGCAAC-3') was designed against the conserved capsid sequences of MNV-1 (GenBank accession No. AY228235), MNV-2 (DQ23041), MNV-3 (DQ223042), and MNV-4 (DQ223043). RTPCR was performed as previously described [7].

\section{Quantitative RT-PCR}

Viral RNA was prepared from the reaction mixture using QIAamp Viral RNA Mini Kits. A forward primer (5'-CAGATCACATGCTTCCCACAT-3'), reverse primer (5'-CCAGAGACCACAAAAGACTCATCA-3') and probe (5'FAM -CCCATTCAACTCCCTCTTCTTGA3'TAMRA) were designed against the conserved sequences of MNV. Quantitative RT-PCR was performed using an EZ RT-PCR Kit (Applied Biosystems, Foster City, CA, USA) and ABI Prism 7700 Sequence Detection System (Applied Biosystems). The following RT-PCR parameters were used: $50^{\circ} \mathrm{C}$ for $2 \mathrm{~min}, 60^{\circ} \mathrm{C}$ for $30 \mathrm{~min}$, $95^{\circ} \mathrm{C}$ for $5 \mathrm{~min}$, and then 50 cycles of denaturation $\left(94^{\circ} \mathrm{C}\right.$, $15 \mathrm{~s})$, annealing $\left(60^{\circ} \mathrm{C}, 30 \mathrm{~s}\right)$, and extension $\left(72^{\circ} \mathrm{C}, 30\right.$ s). The standard curve was generated with serial dilutions of MNV-1 RNA encoding the capsid region synthesized by an AmpliScribe T7 Transcription Kit (Epicentre, Madison, WI, USA).

\section{Sequence analysis of viral RNA}

Viral RNA was purified from MNV-infected RAW cells with a Purelink Viral RNA/DNA Kit (Invitrogen), and cDNA was synthesized using SuperScript III reverse transcriptase (Invitrogen) and an oligo (dT) primer. Genome-specific sequences were amplified with an Expand Long kit (Roche Applied Science, Indianapolis, IN, USA), and PCR products were sequenced directly with a Taq DyeDeoxy Terminator cycle sequencing kit (Applied Biosystems) on an ABI 3730XL DNA analyzer using MNV1-specific primers. When these primers failed, additional sequence-specific primers were designed and used for sequencing. The termini of the MNV genomes were obtained using GeneRacer (Invitrogen), and sequencing was carried out with sequence-specific primers. The nucleotide and protein sequences of ORF1, ORF2, or ORF3 were aligned using BioEdit (http://www. mbio.ncsu.edu/BioEdit/bioedit.html) and employed for subsequent phylogenetic analyses. A phylogenetic tree with 1,000 bootstrap replications was constructed by the neighbor-joining method. The distance of nucleotide substitutions per site was calculated by Kimura's twoparameter method and was visualized using the NJplot software (http://pbil.univ-lyon1.fr/software/njplot.html). Nucleotide and amino acid sequences were analyzed with GENETYX-MAC version 12.2.6 (Genetyx, Tokyo, Japan).

\section{Nucleotide sequence accession number}

The MNV nucleotide sequence determined in this study was deposited in DDBJ under the accession number AB601769.

\section{Results}

Isolation and genetic characterization of a novel MNV

To identify MNV-infected mice, fecal samples were collected from eight mice, and RT-PCR was performed using a novel set of primers designed from conserved sites on MNV genomes. The MNV gene was detected in all eight samples (Fig. 1A). A plaque became visible after nine passages with RAW264.7 cells (Fig. 1B). After two rounds of plaque purification, MNV was isolated as a single plaque and designated as MNV MT30-2. The complete nucleotide sequence was determined, and homology searches with other MNV strains showed the MNV MT30-2 strain to be genetically similar to MNV CR3/2005/USA [15] (Fig. 2). 
A

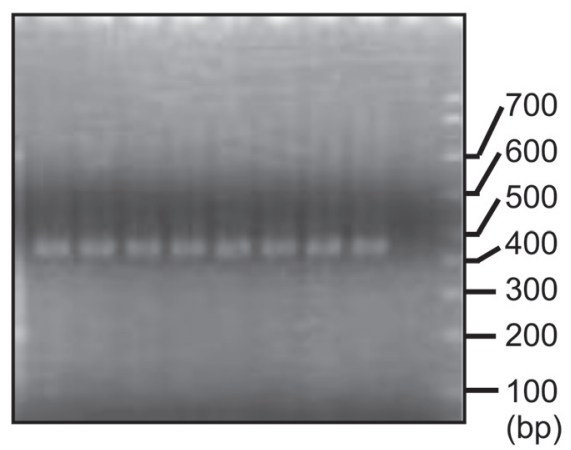

B

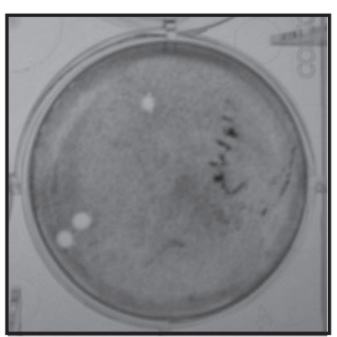

Fig. 1. Detection of MNV gene and MNV plaques. (A) Agarose gel electrophoresis of MNV-specific RT-PCR products (421 bp). (B) Plaques of RAW264.7 cell-adapted MNV.

Table 1. Virucidal effect of PVP-I and $\mathrm{NaOCl}$ compared by real-time RT-PCR and plaque assay

\begin{tabular}{lccc}
\hline Disinfectant & $\begin{array}{c}\text { Treatment time } \\
(\mathrm{s})\end{array}$ & $\begin{array}{c}\text { RNA copy no. } \\
(\log \text { copy no./ml) }\end{array}$ & $\begin{array}{c}\text { Plaque no. } \\
(\log \text { PFU/ml) }\end{array}$ \\
\hline $1 \%(\mathrm{w} / \mathrm{v}) \mathrm{PVP}-\mathrm{I}$ & 0 & 6.69 & 6.38 \\
& 60 & 6.65 & $<2.0$ \\
$0.2 \%(\mathrm{w} / \mathrm{v}) \mathrm{NaOCl}$ & 0 & 6.66 & 6.13 \\
& 60 & 6.30 & 3.13 \\
\hline
\end{tabular}

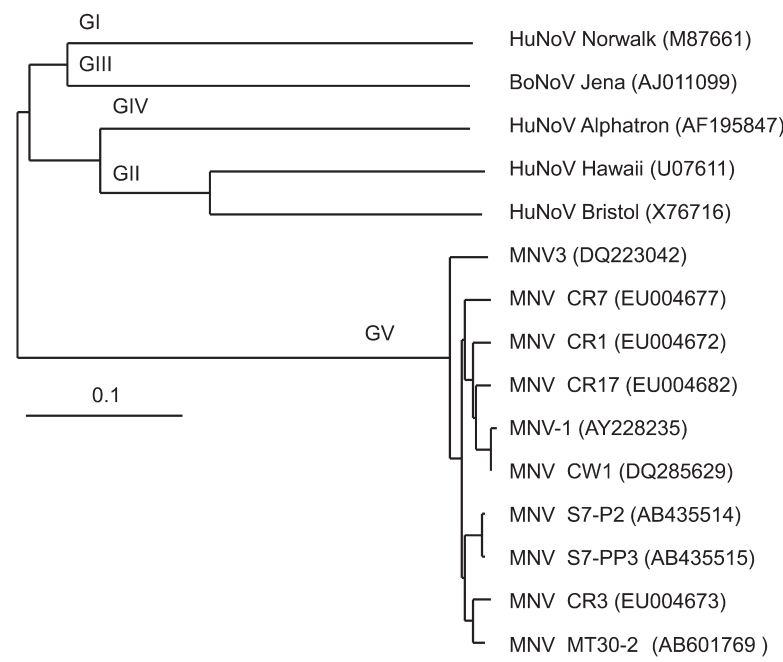

Fig. 2. Phylogenic tree of the Noroviruses. The phylogenic tree was generated using the neighbor-joining method on the basis of an alignment of the entire amino acid sequence of the capsid genes. Numbers in parentheses indicate GenBank accession Nos.

\section{Virucidal activity of antiseptics against $M N V$}

To determine the efficacy of different antiseptics (PVPI, $\mathrm{NaOCl}, \mathrm{BEC}$, and $\mathrm{CHG}$ ) against the MT30-2 strain, we determined the virus titer using a plaque assay after each antiseptic was added to the virus. Both 0.2 and $1 \%$ (w/v) PVP-I reduced the titer by $4 \log _{10}$ within $15 \mathrm{~s}$ (Fig. $3 \mathrm{~A})$. Both 0.1 and $0.2 \%(\mathrm{w} / \mathrm{v}) \mathrm{NaOCl}$ reduced the titer by 1.6 and $3 \log _{10}$ in $60 \mathrm{~s}$, respectively (Fig. 3B). In contrast, $0.1 \%(\mathrm{w} / \mathrm{v}) \mathrm{BEC}$ and $0.5 \%$ (w/v) $\mathrm{CHG}$ resulted in only a slight reduction in the titer after $60 \mathrm{~s}$ of treatment (Fig. 3C). These results indicated that PVP-I and $\mathrm{NaOCl}$ efficiently and rapidly inactivated MNV, whereas both BEC and CHG were ineffective against MNV.

\section{Relationship between plaque assay and quantitative RT- PCR}

The effectiveness of antiseptics against a noncultivatable virus is often measured by quantitative RT-PCR. Thus, we quantified MNV MT30-2 RNA copies in samples treated for $60 \mathrm{~s}$ with $1 \%(\mathrm{w} / \mathrm{v})$ PVP-I or $0.2 \%$ $(\mathrm{w} / \mathrm{v}) \mathrm{NaOCl}$ and compared the values with the virus titers. We found the number of MNV MT30-2 RNA copies was only slightly reduced when the virus titer was greatly reduced (Table 1), i.e., the quantitative RT-PCR results did not necessarily reflect the plaque assay results. 


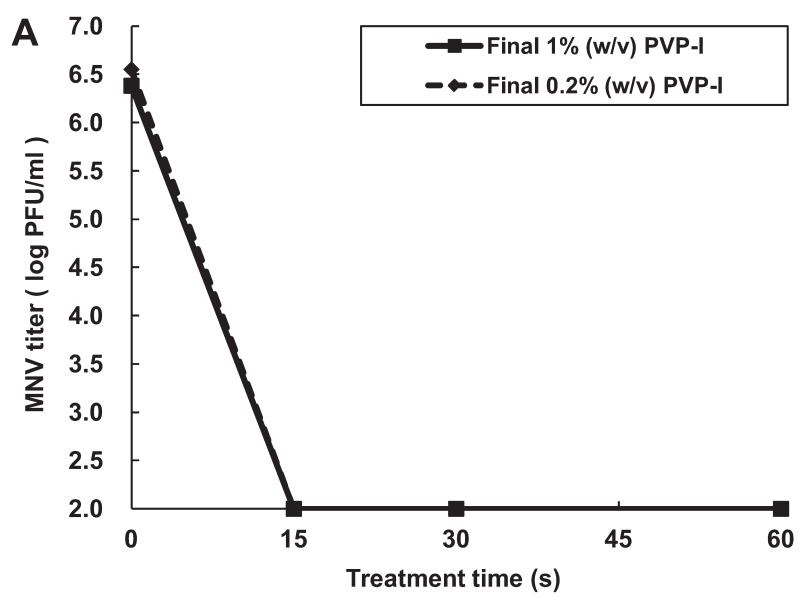

These results coincided with those in a previous report using MNV CW1 [1].

\section{Discussion}

In the present study, we described the isolation of a novel MNV, strain MT30-2. Sequence analysis showed that MT30-2 was genetically similar to MNV CR3/2005/ USA (Fig. 2). Using the MT30-2 strain, we showed that two antiseptics (PVP-I and $\mathrm{NaOCl}$ ) were able to inactivate the virus at low concentrations and in a short contact time (Fig. 3A and 3B). The other two antiseptics, BEC and $\mathrm{CHG}$, which showed virucidal activity against several human viruses [10], appeared to be ineffective against MNV (Fig. 3C). PVP-I and $\mathrm{NaOCl}$ also showed strong virucidal activity against MNV CW1 [1], a genetically separated strain from our work (Fig. 2), suggesting that these two antiseptics potentially possess virucidal activity against various MNV strains. $\mathrm{EtOH}$ also reduced the MNV infectious titer [1]. However, previous reports demonstrated that $\mathrm{EtOH}$ did not show virucidal activity over a short time frame against Feline calicivirus (FCV), another antiseptics-resistant virus that, like MNV, belongs to the Caliciviridae family [4, 11]. On the other hand, PVP-I and $\mathrm{NaOCl}$ are effective for FCV $[3,4,11,16]$. Accordingly, PVP-I and $\mathrm{NaOCl}$ might be effective against not only other MNV strains but also other related noroviruses including HuNoV.

Since the infectivity of $\mathrm{HuNoV}$ is very strong, only a few viral particles induce acute gastroenteritis $[2,14]$. Because it is thought that MNV also has strong infectivity the virus needs to be inactivated as completely possible to prevent the spread of MNV. PVP-I, which showed strong virucidal activity in a short time frame against MNV, would be useful for preventing MNV transmissions by human hand washing in laboratories.

In this study, we found that the results of quantitative RT-PCR were not consistent with those of the virucidal assay (Table 1), and the detection of viral RNA was not necessarily related to the virus titers, demonstrating the importance of conducting infectivity assays when evaluating the efficacy of antiseptics. The efficacy of antiseptics may be underestimated if quantitative RT-PCR is used for evaluation, as reported in a previous paper [1]. Thus, interpretation of the results of quantitative respectively. 
RT-PCR needs further consideration.

In conclusion, our data demonstrate that a novel MNV isolate, MT30-2, is a useful tool to evaluate the effectiveness of antiseptics and that PVP-I as well as $\mathrm{NaOCl}$ is the most effective antiseptics for disinfection and infection control of MNV.

\section{References}

1. Belliot, G., Lavaux, A., Souihel, D.,Agnello, D., and Pothier, P. 2008. Use of murine norovirus as a surrogate to evaluate resistance of human norovirus to disinfectants. 2008. Appl. Environ. Microbiol. 74: 3315-3318.

2. Cremon, C., De Giorgio, R., and Barbara, G. 2010. Norovirus gastroenteritis. N. Engl.J. Med. 361: 1776-1785.

3. Doultree, J.C., Druce, J.D., Birch, C.J., Bowden, D.S., and Marshall, J.A. 1999. Inactivation of feline calicivirus, a Norwalk virus surrogate. J. Hosp. Infect. 41: 51-57.

4. Duizer, E., Bijkerk, P., Rockx, B., De Groot, A., Twisk, F., and Koopmans, M. 2004. Inactivation of caliciviruses. Appl. Environ. Microbiol. 70: 4538-4543.

5. Duizer, E., Schwab, K.J., Neill, F.H., Atmar, R.L., Koopmans, M.P., and Estes, M.K. 2004. Laboratory efforts to cultivate noroviruses. J. Gen. Virol. 85: 79-87.

6. Goto, K., Hayashimoto, N., Yasuda, M., Ishida, T., Kameda, S., Takakura, A., and Itoh, T. 2009. Molecular detection of murine norovirus from experimentally and spontaneously infection mice. Exp. Anim. 58: 135-140.

7. Hsu, C.C., Riley, L.K., Wills, H.M., and Livingston, R.S. 2006. Persistent infection with and serologic cross-reactivity of three novel murine noroviruses. Comp. Med. 56: 247251.

8. Hsu, C.C., Wobus, C.E., Steffen, E.K., Riley, L.K., and Livingston, R.S. 2005. Development of a microsphere-based serologic multiplexed fluorescent immunoassay and a reverse transcriptase PCR assay to detect murine norovirus 1 infection in mice. Clin. Diagn. Lab. Immunol. 10: 11451151 .

9. Karst, S.M., Wobus, C.E., Lay, M., Davidson, M., and Virgin, H.W. 2003. STAT 1-dependent innate immunity to a Norwalk-like virus. Science 299: 1575-1578.

10. Kawana, R., Kitamura, T., Nakagomi, O., Matsumoto, I., Arita, M., Yoshihara, N., Yanagi, K., Yamada, A., Morita, O., Yoshida, Y., Furuya, Y., and Chiba, S. 1997. Inactivation of human viruses by povidone-iodine in comparison with other antiseptics. Dermatology 195: 29-35.

11. Lages, S.L., Ramakrishnan, M.A., and Goyal, S.M. 2008. In-vivo efficacy of hand sanitisers against feline calicivirus: a surrogate for norovirus. J. Hosp. Infect. 68: 159-163.

12. Maler, M. and Kol, W. 2009. A serological survey to evaluate contemporary prevalence of viral agents and Mycoplasma pulmonis in laboratory mice and rats in western Europe. Lab. Anim. (NY) 38: 161-165.

13. Mumphrey, S.M., Changotra, H., Moore, T.N., HeimannNichols, E.R., Wobus, C.E., Reilly, M.J., Moghadamfalahi, M., Shukla, D., and Karst, S.M. 2007. Murine norovirus 1 infection is associated with histopathological changes in immunocompetent hosts, but clinical disease is prevented by STAT1-dependent interferon responses. J. Virol. 81: 3251-3263.

14. Teunis, P.F., Moe, C.L., Liu, P., Miller, S.E., Lindesmith, L., Baric, R.S., Le Pendu, J., and Calderon, R.L. 2008. Norwalk virus: how infectious is it? J. Med. Virol. 80: 1468-1476.

15. Thackray, L.B., Wobus, C.E., Chachu, K.A., Liu, B., Alegre, E.R., Henderson, K.S., Kelley, S.T., and Virgin, H.W. 2007. Murine noroviruses comprising a single genogroup exhibit biological diversity despite limited sequence divergence. $J$. Virol. 81: 10460-10473.

16. Urakami, H., Ikarashi, K., Okamoto, K., Abe, Y., Ikarashi, T., Kono, T., Konagaya, Y., and Tanaka, N. 2007. Chlorine sensitivity of feline calicivirus, a norovirus surrogate. Appl. Environ. Microbiol. 73: 5679-5682.

17. Wobus, C.E., Thackray, L.B., and Virgin, H.W. 2006. Murine norovirus: a model system to study norovirus biology and pathogenesis. J. Virol. 80: 5104-5112.

18. Wobus, C.E., Karst, S.M., Thackray, L.B., Chang, K.O., Sosnovtsev, S.V., Belliot, G., Krug, A., Mackenzie, J.M., Green, K.Y., and Virgin, H.W. 2004. Replication of norovirus in cell culture reveals a tropism for dendritic cells and macrophages. PLoS Biol. 2: 2076-2084. 